\title{
Topical anaesthesia with oxybuprocaine versus sub-Tenon's infiltration with $2 \%$ lignocaine for small incision cataract surgery
}

H B Chittenden, W R Meacock, J A A Govan

\begin{abstract}
Aims-To determine whether topical anaesthesia in small incision self-sealing phacoemulsification cataract surgery provides comparable anaesthesia to subTenon's infiltration.

Methods-Thirty five patients undergoing small incision self-sealing phacoemulsification cataract surgery were allocated randomly to receive topical anaesthesia with $0.4 \%$ oxybuprocaine or sub-Tenon's infiltration with $2 \%$ lignocaine. Pain experienced during the operation was assessed by asking the patient to score on a visual analogue graphic pain score chart.

Results-The median pain score for the topical group (3) was significantly higher than that of the sub-Tenon's group $(0)(p=$ 0.004).

Conclusion-Sub-Tenon's infiltration is superior to topical anaesthesia in ensuring patient comfort during small incision scleral tunnel self-sealing phacoemulsification cataract surgery.

(Br f Ophthalmol 1997;81:288-290)
\end{abstract}

Over the past 2 years, small incision self-sealing phacoemulsification cataract surgery has been performed on more than 600 patients by one surgeon (JAAG), using sub-Tenon's infiltration as the preferred method of local anaesthesia. This technique avoids many of the risks of retrobulbar and peribulbar local anaesthetic injections, yet provides good anaesthesia with variable akinesia. ${ }^{1}$ Topical anaesthesia has also been advocated as a safer local anaesthetic technique compared with retrobulbar and peribulbar anaesthesia, providing effective intraoperative anaesthesia, although no akinesia. ${ }^{23}$ The obvious advantages of topical anaesthesia versus sub-Tenon's infiltration are time saved in administering the local anaesthetic and shorter duration of action, which allows the patient to regain visual acuity rapidly after surgery. ${ }^{2}$ Another potential advantage of topical anaesthesia versus sub-Tenon's infiltration is less patient discomfort associated with administration of the local anaesthetic. However, from personal experience, most of the discomfort associated with sub-Tenon's infiltration is ascribed to instillation of the anaesthetic drops.

In deciding whether to change from subTenon's anaesthesia to topical anaesthesia, patient comfort during the operative procedure was deemed to be the most important consid- eration. A study was thus undertaken to assess the pain experienced by individual patients during small incision self-sealing phacoemulsification cataract surgery performed under topical anaesthesia compared with subTenon's infiltration.

\section{Materials and methods}

Thirty five patients undergoing small incision self-sealing phacoemulsification cataract surgery were allocated randomly to receive topical or sub-Tenon's anaesthesia. Topical anaesthesia was provided by $0.4 \%$ oxybuprocaine hydrochloride (Benoxinate), instilled onto the cornea and conjunctiva. For the sub-Tenon's technique, Benoxinate drops were instilled as for topical anaesthesia. A small subconjunctival injection of 2\% lignocaine with 1:200 000 adrenaline was then given in the inferior-nasal quadrant approximately $5 \mathrm{~mm}$ from the limbus, in order to raise a small bleb, and a small incision made in the conjunctiva with Wescott's spring scissors. The scissors were then used to blunt dissect deep to the Tenon's capsule to bare sclera. A blunt 19 gauge curved cannula was then used to deliver approximately $2-3 \mathrm{ml}$ of $2 \%$ lignocaine with 1:200 000 adrenaline to the posterior sub-Tenon's potential space.

A superior rectus fixation suture was used in the sub-Tenon's group but not in the topical group. The operative technique for the two groups was otherwise identical and performed by one surgeon (JAAG). A limbal peritomy was made at the right upper quadrant and light cautery applied to the scleral vessels. A scleral tunnel was fashioned and the anterior chamber entered in clear cornea. The anterior chamber was then filled with sodium hyaluronate (Healon) and a one step capsulorrhexis was performed followed by hydrodissection at the cortico-capsular interface. Phacoemulsification was followed by further injection of viscoelastic into the anterior chamber and capsular bag. The scleral tunnel was enlarged and a $5.5 \mathrm{~mm}$ PMMA intraocular lens implant (IOL) inserted through it into the capsular bag. The IOL was dialled into position and the viscoelastic aspirated. The conjunctiva was closed by manual apposition or saline bleb. No sutures were used in any of the cases. Chloramphenicol drops $(0.5 \%)$ were instilled at the end of the procedure and the eye covered with a clear plastic shield.

No premedication was used and no sedation or other analgesia was required during surgery.
Accepted for publication 13 January 1997 
Table 1 Number of patients feeling pain or discomfort during a particular stage of the operation

\begin{tabular}{lll}
\hline & Sub-Tenon's & Topical \\
\hline Superior rectus fixation suture & 3 & 6 \\
Cautery & & 1 \\
Phacoemulsification & 1 \\
Enlargement of scleral tunnel & 2 \\
Insertion of IOL & \\
Conjunctival closure (by saline & 1 \\
$\quad$ bleb) & \\
\hline
\end{tabular}

During the operation a healthcare assistant sat beside the patients holding their right hand. Patients were instructed to squeeze the healthcare assistant's hand if at any time they felt any pain. When the patients indicated that they were in pain, the particular stage of the operation was noted. On the first postoperative day, each patient was shown a $10 \mathrm{~cm}$ visual analogue graphic pain score chart, with a numerical and descriptive rating scale, graded from $0-10$. Patients were invited to score where on the chart they could grade any discomfort or pain experienced during the operation. Any particular comments relating to the type of anaesthetic used or the operation itself were also recorded at this time.

\section{Results}

Thirty five patients had small incision selfsealing phacoemulsification cataract surgery, of which 16 procedures were performed under topical anaesthesia and 19 after sub-Tenon's infiltration.

Three patients in the sub-Tenon's group indicated that they were in pain during the operation by squeezing the healthcare assistant's hand. In each case the pain occurred only once, during insertion of the superior rectus fixation suture. By comparison, seven patients in the topical group indicated that they were in pain during the operation, four of these on more than one occasion. Pain during cautery of the scleral vessels was reported most frequently (Table 1).

The subjective assessment of pain experienced by the patients during the operation is represented in Figure 1 by a pain score chart. The median pain score for the topical group

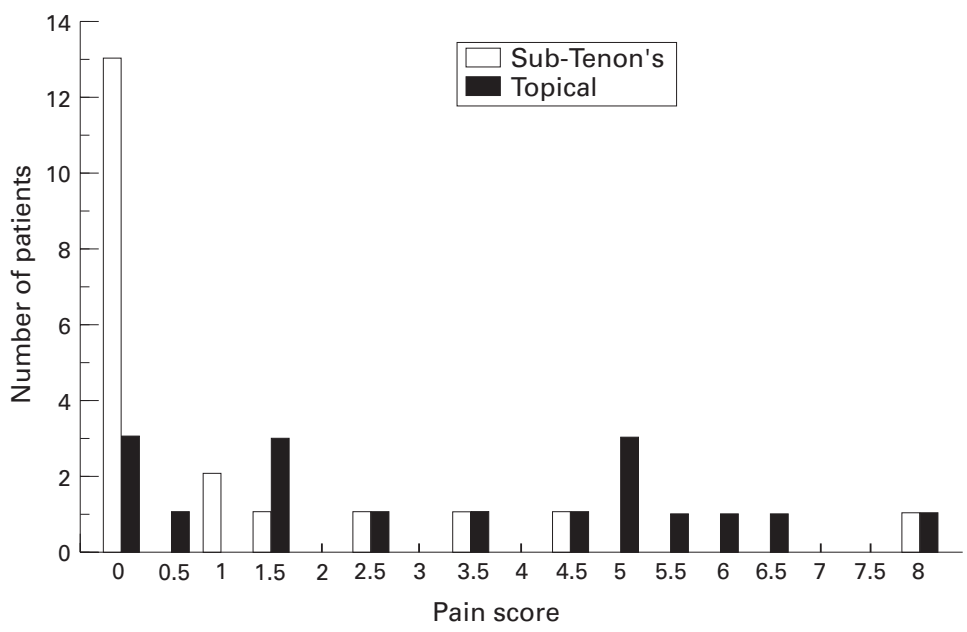

Figure 1 Pain experienced during operation.
(3) was significantly higher than that of the sub-Tenon's group (0) (Mann-Whitney U test, $\mathrm{p}=0.004)$. Three patients in the topical group were having their second eye operated on. Each reported that they experienced more discomfort under topical anaesthesia than they remembered experiencing during their first cataract operation, which was performed under sub-Tenon's local anaesthetic.

\section{Discussion}

Topical anaesthesia and sub-Tenon's infiltration are both accepted methods of providing local anaesthesia for small incision self-sealing phacoemulsification cataract surgery. Although topical anaesthesia is less invasive and quicker to administer than sub-Tenon's infiltration, acceptable cataract surgery under local anaesthesia depends to a large extent on the patient's comfort during the procedure.

It became apparent quite early on in the study that patients were much more comfortable during the operation when sub-Tenon's infiltration was employed compared with topical anaesthesia. For this reason it was felt inappropriate to continue with topical anaesthesia and the study was therefore stopped after 35 patients. It was initially intended to include at least 50 patients in the study.

Patients in the sub-Tenon's group only experienced pain when the superior rectus fixation suture was inserted. A fixation suture may be necessary when sub-Tenon's infiltration is used, particularly when depression of the globe is inadequate in a sunken eye. This surgeon (JAAG) now inserts a $7 / 0$ silk peripheral corneal fixation suture when necessary. A fixation suture is not required when topical anaesthesia is used as a cooperative patient is able to look down easily. Although patients in the topical group generally found the whole procedure more uncomfortable, particular pain was felt during cautery of the scleral vessels. Topical anaesthesia may therefore be more appropriate when cautery is not required, such as in phacoemulsification through a clear corneal incision.

In another study, which compared topical and sub-Tenon's anaesthesia, the topical anaesthesia was supplemented with a subconjunctival injection of $2 \%$ lignocaine, $2 \mathrm{~mm}$ posterior to the superior limbus, to facilitate painless cautery of the scleral vessels. ${ }^{4}$ In cases where a scleral tunnel is preferable to a corneal incision (for example, when subsequent penetrating keratoplasty may be necessary) supplementing the topical anaesthesia with a subconjunctival injection of $2 \%$ lignocaine may be helpful. A combination of topical anaesthesia and subconjunctival injection of lignocaine has also been used for extracapsular cataract extraction. $^{5}$

With topical anaesthesia preserved downgaze allows greater access for a scleral tunnel incision. However, the lack of akinesia necessitates the constant use of a second instrument to immobilise the eye, particularly during the capsulorrhexis. Although this is less of a problem for the experienced surgeon, this study found that the inferior anaesthesia provided by 
the topical method made the operative procedure more stressful for both patient and surgeon, and technically more demanding for the surgeon.

The quality of anaesthesia of different topical agents may vary in small incision cataract surgery, but no study appears to have compared the effectiveness of all the available topical anaesthetics. Although amethocaine tends to be used in the UK (tetracaine in the USA), ${ }^{2}{ }^{6}$ there is no evidence to suggest that either of these two agents is more effective than oxybuprocaine when used for topical anaesthesia in small incision cataract surgery. In addition, oxybuprocaine has the advantage of being less irritant to the corneal epithelium and the conjunctiva than both tetracaine and amethocaine. $^{78}$

This study has shown that sub-Tenon's infiltration is superior to topical anaesthesia in ensuring patient comfort during small incision scleral tunnel self-sealing phacoemulsification cataract surgery and for this reason remains the authors' preferred choice of safe, minimally invasive local anaesthesia.

1 Stevens JD. A new local anaesthetic technique for cataract extraction by one quadrant sub-Tenon's infiltration. $\mathrm{Br} \mathcal{F}$ Ophthalmol 1992;76:670-4.

2 Kershner RM. Topical anaesthesia for small incision self-sealing cataract surgery. I Cataract Refract Surg 19:290-2.

3 Grabow HB. Topical anaesthesia for cataract surgery. Eur $\mathcal{F}$ Implant Ref Surg 1993;5:20-3.

4 Manners TD, Burton RL. Randomised trial of topical versus sub-Tenon's local anaesthetic for small incision cataract surgery. Eye 1996;10:367-70.

5 Smith R. Cataract extraction without retrobulbar anaesthetic injection. Br f Ophthalmol 1990;74:205-7.

6 Duguid IG, Clauoe CMP, Thamby-Rajah Y, Allan BD, Dart $\mathrm{JK}$, Steele AD. Topical anaesthesia for phacoemulsification surgery. Eye 1995;9:456-9.

7 Sclegel HE, Swan KC. Benoxinate (dorsacaine) for rapid corneal anaesthesia. Arch Ophthalmol 1954;51:663-70.

8 Gilmam AG, Goodman LS, Rall TW, Murad R. The pharmacological basis of therapeutics. 7th ed. London: Collier-MacMillan, 1985:302-12. 